\title{
Novel Combination of Mesenchymal Stem Cell-Conditioned Medium with Sorafenib Have Synergistic Antitumor Effect of Hepatocellular Carcinoma Cells
}

\author{
Iman Seyhoun ${ }^{1}$, Saieh Hajighasemlou ${ }^{1,2 *}$, Jafar Ai ${ }^{1}$, Faezeh Hosseinzadeh ${ }^{1}$, Milad \\ Mirmoghtadaei $^{3}$, Seyed Moein Seyhoun ${ }^{4}$, Benyamin Parseh ${ }^{1}$, Shahrokh Abdolahi ${ }^{1}$, \\ Zeinab Ghazvinian ${ }^{1}$, Mahdi Shadnoush ${ }^{5}$, Javad Verdi ${ }^{1 *}$
}

\begin{abstract}
Objective: Hepatocellular carcinoma (HCC) is the most common liver malignancy. Sorafenib is the first-line systemic treatment for advanced HCCs. However, due to safety concerns, researchers are now looking for ways to boost the efficacy of the medication. One approach for reducing toxicity is combining sorafenib with other agents so that a lower dose of sorafenib is required. Mesenchymal stromal cells (MSCs) can have an inhibitory effect on HCC tumor growth. Mesenchymal Stem Cell-Conditioned Medium (MSC-CM) is the substance extracted from MSC culture and contains most of the potential cytokines secreted by MSCs. We, therefore, anticipated a synergistic Antitumor Effect of sorafenib in Combination with MSC-CM. In this study, we used HepG2 as our target cell lines. Methods: HepG2 cells were treated with sorafenib alone and with sorafenib + MSC-CM. CCK-8 assay was used to evaluate and compare the inhibition of cell growth between the two groups with different treatments. Results: The combination treatment of cell lines with sorafenib and MSC-CM had significantly reduced the values of IC50 compared to the use of sorafenib alone (3.4 vs. 2.7 respectively). Conclusion: This study suggests that a combination of sorafenib with MSC-CM can synergistically suppress the growth of HCC cells.
\end{abstract}

Keywords: Mesenchymal stem cell- sorafenib- hepatocellular carcinoma- HepG2

Asian Pac J Cancer Prev, 20 (1), 263-267

\section{Introduction}

Hepatocellular carcinoma (HCC) is the fifth most common cancer and the second leading cause of cancer-related mortalities worldwide (Hajighasemlou et al., 2018; Sadaf et al., 2018). There are many methods in the treatment of HCC such as chemotherapy by using new antitumor drugs, operation, intervene therapy, liver transplantation (LT), and so on. Liver resection is still the mainstay of treatment for $\mathrm{HCC}$ and provides the consistent long-term survival. However, the resectability is limited by tumor extent, location, or underlying liver dysfunction, with only a minority of HCC being potentially resectable. All these leave LT rather than liver resection as the only potentially curative option, which increase the possibilities of HCC resection for patients with nonresectable tumor or severe hepatic failure. It is reported that the 5-year survival for HCC patients undergoing LT has been steadily improved from $25.3 \%$ in 1987 to $61.1 \%$ during the most recent period studied. Despite the total hepatectomy and liver replace, recurrence and metastasis remained the major obstacles to more prolonged survival after LT for HCC (Yoo et al., 2003). Thereby, novel therapeutic strategies to prevent recurrence after LT are needed. sorafenib is the first targeted therapy that was approved for use in advanced HCC. It exerts its effects by inducing tumor cell apoptosis and reducing tumor angiogenesis (Liu et al., 2006; Strumberg et al., 2007; Almhanna and Philip, 2009; Iijima et al., 2011). The unfavorable side effect profile of the drug with minimal response rates have prompted researchers to look for new alternatives, including combining sorafenib with other potential agents to reduce the dosage and improve its efficacy (Carr et al., 2010; Hosseinzadeh et al., 2018).

Mesenchymal stem cells (MSCs) are multipotent stem cells that are present in various tissues, including placenta, bone marrow and adipose tissues (Jahan et al., 2017). Due to their tropism to tumor sites and their ability

${ }^{1}$ Tissue Engineering and Applied Cell Sciences, ${ }^{3}$ School of Medicine, Tehran University of Medical Sciences, ${ }^{2}$ Food and Drug Control Laboratory (FDCL), Iran Ministry of Health and Medical Education, ${ }^{5}$ Nutrition and Food Technology, Shahid Beheshti University of Medical Sciences, Tehran, ${ }^{4}$ Department of Microbiology, Qom Branch, Islamic Azad University, Qom, Iran, *ForCorrespondence: S_ghasemlou@yahoo.com,Javad0verdi@gmail.com 
to suppress tumor growth, MSCs are considered potential candidates for cancer therapy (Peng et al., 2014; Sage et al., 2016). Several studies have demonstrated antitumor properties for MSCs on different malignancies . Cho et al., (2009) characterized an inhibitory effect for CM-MSCs on ovarian tumor cells (SK-OV-3) which is likely through a downregulation of insulin-like growth factors, IL8 and VEGF. Atsuta et al. has also provided proof that MSCs can inhibit the proliferation of multiple myeloma cells through Fas/Fas-L pathway.

All the past investigations however, only revolve around the use of MSCs and sorafenib alone and to our knowledge, the effect of these two agents in combination has not been investigated on HCC. Here we have hypothesized that MSC-CM can augment the effect of sorafenib on suppression of tumor growth.

\section{Materials and Methods}

\section{Reagents}

Sorafenib was purchased from American LC LAB company. Human bone marrow mesenchymal stem cells (BM-MSC) and human hepatocellular carcinoma cell lines (HepG2) were purchased from Iranian Biological Resource Center (IBRC). HepG2 is an immortalized cell line consisting of human liver carcinoma cells, derived from the liver tissue of a 15-year-old Caucasian male who had a well-differentiated hepatocellular carcinoma.

\section{Cell culture}

Human bone marrow mesenchymal stem cells (hMSC) and Human hepatocellular carcinoma cell lines (HepG2) were cultured in high glucose DMEM media supplemented with $10 \%$ fetal bovine serum, streptomycin $(100 \mu \mathrm{g} / \mathrm{ml})$, penicillin $(100 \mathrm{U} / \mathrm{ml})$, in standard conditions of incubator at $37^{\circ} \mathrm{C}$ in a $5 \% \mathrm{CO}_{2}$ atmosphere and $95 \%$ humidity.

\section{Preparation of Conditioned Medium}

Conditioned medium was collected by filtering through a $0.22 \mu \mathrm{m}$ membrane when the confluency of MSCs reached about $70 \%$ to $80 \%$. After filtration, condition media were mixed with fresh media to yield different percentages.

\section{CCK-8 assay of sorafenib}

The cell counting kit-8 assay (Molecular Devices, Japan) was used to determine cell viability. Various cell densities were seeded in 96 well microplates in triplets. CCK-8 reagent was added to each well at the same time on consecutive days after treatment of HepG2. After incubation of the plates for $2 \mathrm{~h}$ at $37^{\circ} \mathrm{C}$, absorbance at $490 \mathrm{~nm}$ was measured using a microplate. Results were expressed as a percentage of control at the completion of each incubation period. Control groups were treated with fresh media.

\section{Synergy experiments}

Synergy between sorafenib and MSC-CM was assessed in HCC cell lines in vitro. Cells were incubated with each agent separately and in combination for $48 \mathrm{~h}$ before assessment of cytotoxicity by CCK-8 assay as described above. Cells were plated as for CCK- 8 assays and treated for $48 \mathrm{~h}$ with different concentrations of sorafenib and MSC-CM, alone and in combination. The half-maximal (50\%) inhibitory concentration (IC50) of each drug constituted the median value within the chosen concentration range. Combination index (CI) plots were generated using the CalcuSyn (v2.0) software (Biostat, Cambridge, UK) based on algorithms developed by Chou and Talalay to determine whether the drugs synergize. Based on this approach, CI values $<0.9$ are considered synergistic, values $>1.1$ are antagonistic, and values of 0.9-1.1 are approximately additive.

\section{Statistical analysis}

Results are presented as mean \pm SD. Differences between the two groups were calculated using Student's t-test. $P$ values under 0.05 were considered significant.

\section{Results}

Inhibitory Effect of MSC-Conditioned medium on HCC cell growth

Effects of MSC-CM on HepG2 cells after $72 \mathrm{~h}$ were determined using CCK-8 assay. All groups showed a significant reduction in the proliferative capacity of HepG2 cells compared to the control group $(\mathrm{p}<0.001)$ (Figure 1). Inhibitory Effect of sorafenib alone on HCC cell growth

The inhibitory effect of sorafenib on HepG2 cell growth was evaluated by using the Cell Counting Kit-8 (CCK-8) at different doses. Determination of half-maximal $(50 \%)$ inhibitory concentration (IC50) of sorafenib was calculated by nonlinear regression analysis using Excel software and was equal to $3.4 \pm 0.09 \mu \mathrm{M}$ (Figure 2).

Inhibitory Effect of sorafenib in combination with MSCCM on HCC cell growth

ACI approach was used to determine whether these molecules caused additive or synergistic inhibition. When MSC-CM $(20 \%, 40 \%, 60 \%$ or $80 \%)$ was added to cells incubated with increasing doses of sorafenib, the combinatory effect was evident in HepG2 cells (Table 1). For HepG2 cells, the CI values were between 0.75-0.99, indicating a significant synergism between sorafenib and MSC-CM , particularly at high MSC-CM concentrations $(60 \%, 80 \%)$ (Table 1).

We further examined whether the combination of relatively low concentrations of sorafenib with MSC-CM could synergistically inhibit HCC cell growth in-vitro. For this purpose, we used MSC-CM $(80 \%)$. HepG2 cells were treated with MSC-CM $(80 \%)$ in the presence of different

Table 1. The Synergy of Killing by Sorafenib and Celecoxib

\begin{tabular}{lc}
\hline & Combination Index (CI) \\
\hline sorafenib+ MSC-CM (20\%) & $99 \pm 0.4^{*}$ \\
sorafenib+ MSC-CM (40\%) & $94 \pm 0.6^{*}$ \\
sorafenib+ MSC-CM (60\%) & $82 \pm 0.5^{* *}$ \\
sorafenib+ MSC-CM (80\%) & $75 \pm 0.1^{* *}$ \\
\hline
\end{tabular}

*, Addiitive; **, Synergistic 
A

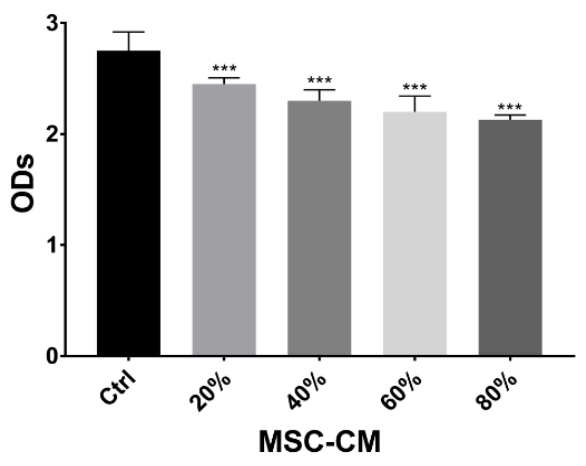

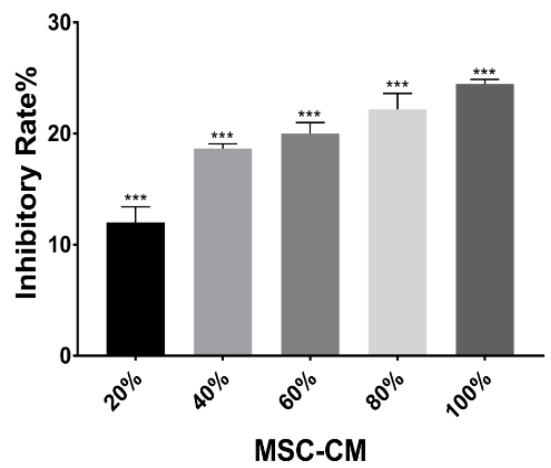

Figure.1. Inhibitory Effects of MSC-CM on HepG2 Cell Growth in vitro. (A)Effects of MSC-CM on HepG2 cells after $72 \mathrm{~h}$ were determined by CCK-8 assay. Results showed that at 20, 40, 60, 80 and 100\% of MSC-CM, the proliferation capacity of HepG2 cells obviously decreased and was significantly different from that of control groups $(* * * p<0.001)$. (b)Inhibition effect analysis of

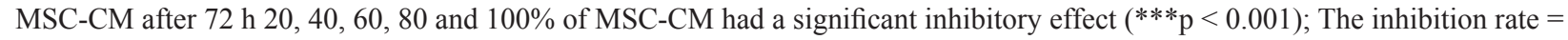
1 - (OD value of treatment group / OD value of control group). Results were shown as mean $\pm \mathrm{SD}, \mathrm{n}=6 .{ }^{* * *} \mathrm{p}<0.001$

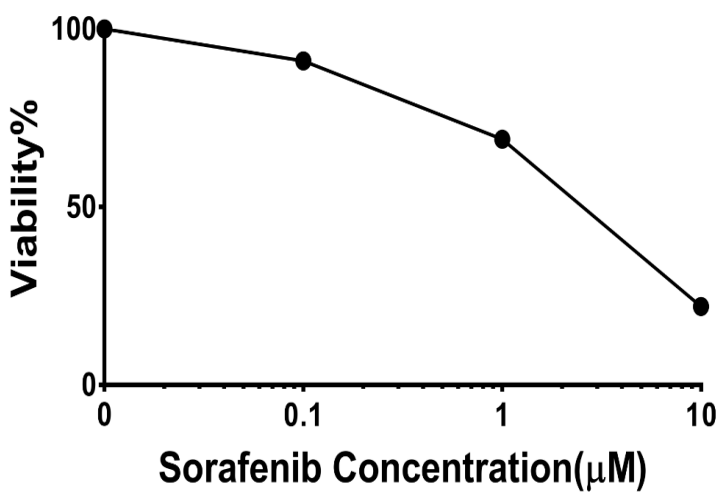

Figure 2. Determination of Half-Maximal (50\%) Inhibitory Concentration (IC50) of Sorafenib-Mediated Cytotoxicity in HCC Cells. Cells were cultured in the presence or absence of sorafenib for $72 \mathrm{~h}$ in DMEM high glucose containing $10 \%$ FBS. treatment of HepG2 cells with increasing concentrations of sorafenib (0.1-10 $\mu \mathrm{mol} / \mathrm{l}$; left) Data are expressed as the mean \pm SEM of five replicates.

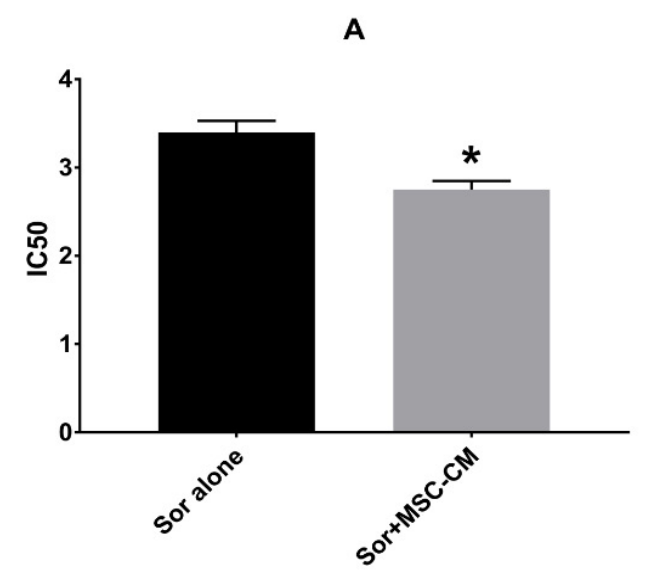

doses of sorafenib $(0,0.01,1$, and $10 \mu \mathrm{mol} / \mathrm{l})$ for $72 \mathrm{~h}$. IC50 in the sorafenib+MSC-CM group was significantly decreased compared to sorafenib alone (Figure 3A).

IC50 of sorafenib was $2.7 \pm 0.07 \mu \mathrm{M}$ when used with MSC-CM (80\%) compared to $3.4 \pm 0.09 \mu \mathrm{M}$ when used alone. The morphologies of HepG2 cells after treatment with sorafenib, MSC-CM80\% and combination of both are shown in Figure 3B.

\section{Discussion}

Hepatocellular carcinoma (HCC) is the most common malignant tumor of hepatocytes. Sorafenib is the first FDA-Approved systemic targeted therapy for the disease (Liu et al., 2006). The side effect profile of sorafenib has been a major concern and has limited the efficacy of the drug (El-Serag et al., 2008); These include: cutaneous toxic effects - rash (19-40\%), mucositis (20\%), xerosis (16\%), alopecia (27\%), and xerostomia (11\%) (Lacouture

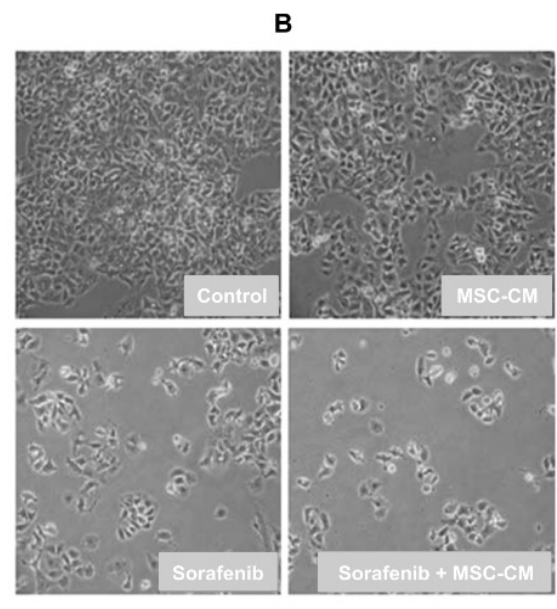

Figure 3. Comparison Effect of Sorafenib Alone VS Sorafenib Plus MSC-CM80\% on Cell Viability of Human Hepatocellular Carcinoma (HCC) Cells. A: Comparation of half-maximal (50\%) inhibitory concentration (IC50) of sorafenib alone and sorafenib with MSC-CM on cell proliferation in HCC cells. HepG2 cells were exposed to different concentrations of sorafenib alone and with MSC-CM80\% for $72 \mathrm{~h}$. Cell viability was assessed by CCK-8 analysis. MSC-CM80\% significantly reduces the IC50 of sorafenib. Data represent the mean \pm standard deviation of five independently-determined IC50 values. ${ }^{*} \mathrm{p}<0.05, \mathrm{C}$; Morphology of HCC (HepG2) cells after treatment with sorafenib, MSC-CM80\% or both. 
et al., 2008). One approach to get around this problem is to use lower doses of the drug in a way where it wouldn't compromise its effectiveness. Mohieldin and et al. showed that sorafenib when combined with Bio-A have synergistic cytotoxicity on HepG2 cells, via inducing apoptosis and interfering with the cell cycle (Youssef et al., 2016). In another study, Ming-Hua and et al. demonstrated that a combination of sorafenib with novel benzimidazole derivatives bearing a pyrrolidine side chain have strong antitumor properties in HCC animal models (Hsu et al., 2017).

In this study we investigated whether adding MSC-CM could boost the antitumor properties of sorafenib in human HCC cells. MSCs can be used to suppress the development of HCC. Their multipotency, immunoregulatory, pro-regenerative effects (Hsu et al., 2017) and their chemotactic properties to home to tumor invaded regions make them a good candidate for cancer therapy (Bayo et al., 2017). MSCs inhibit tumor growth by increasing inflammatory infiltration (Ohlsson et al., 2003), inhibiting angiogenesis (Otsu et al., 2009), suppressing the signaling of Wnt (Qiao et al., 2008a; Qiao et al., 2008b) and AKT (Khakoo et al., 2006), and inducing cell cycle arrest and apoptosis 4 (Cousin et al., 2009; Dasari et al., 2010; Lu et al., 2008).

In this study, we could demonstrate that the cotreatment of sorafenib with MSC-CM (80\%) can augment the cytotoxic effects of sorafenib on the HepG2 cell lines. These results were all deduced from an in-vitro study of HepG2 cells. Future studies could include more extensive in-vivo studies on xenograft models of HCC to further confirm, evaluate the side effects, and better characterize the mechanism behind this effect. This would also examine the rationality of combinational strategies for use at the bedside but this steady need extensive in-vivo study for approved.

In conclusion, the synergy between sorafenib and MSC_CM 80\% in HCC cells is clinically significant and allowing for the use of lower doses of sorafenib with more efficiency than those currently used. This therapeutic strategy needs to more extensive in-vivo studies.

\section{Abbreviations}

HCC: Hepatocellular Carcinoma

MSCs: Mesenchymal Stem Cell-Conditioned Medium

MSC-CM: Mesenchymal stem cells condition media IC50: The half-maximal (50\%) inhibitory

Concentration

CCK-8: Cell Counting Kit-8

CM: Conditioned Media

HFSR: Hand-foot skin reaction

BM-MSC: bone marrow mesenchymal stem cells

IL8: Interleukin 8

VEGF :Vascular endothelial growth factor

Fas-L: Fas ligand

\section{Financial support}

Tehran University of Medical Science provided financial support.

\section{Conflict of interest}

Authors have no conflict of interest to declare.

\section{Acknowledgements}

We would like to thank all our study participants for their commitment to this study.

\section{References}

Almhanna K, Philip PA(2009). Safety and efficacy of sorafenib in the treatment of hepatocellular carcinoma. OncoTargets Ther, 2, 261.

Bayo J, Real A, Fiore EJ, et al (2017). IL-8, GRO and MCP-1 produced by hepatocellular carcinoma microenvironment determine the migratory capacity of human bone marrow-derived mesenchymal stromal cells without affecting tumor aggressiveness. Oncotarget, 8, 80235.

Carr BI, Carroll S, Muszbek N, et al (2010). Economic evaluation of sorafenib in unresectable hepatocellular carcinoma. J Gastroenterol Hepatol, 25, 1739-46.

Cho JA, Park H, Kim HK, et al (2009). Hyperthermia-treated mesenchymal stem cells exert antitumor effects on human carcinoma cell line. Cancer, 115, 311-23.

Cousin B, Ravet E, Poglio S, et al (2009). Adult stromal cells derived from human adipose tissue provoke pancreatic cancer cell death both in vitro and in vivo. PLoS One, 4, e6278.

El-Serag HB, Marrero JA, Rudolph L, et al (2008). Diagnosis and treatment of hepatocellular carcinoma. Gastroenterology, 134, 1752-63.

Dasari VR, Velpula KK, Kaur K, et al (2010). Cord blood stem cell-mediated induction of apoptosis in glioma downregulates X-linked inhibitor of apoptosis protein (XIAP). PLoS One, 5, e11813.

Hajighasemlou S, Pakzad S, Ai J, et al (2018). Characterization and validation of hepatocellular carcinoma (HCC) xenograft tumor as a suitable liver cancer model for preclinical mesenchymal stem cell studies. Asian Pac J Cancer Prev, 19, 1627.

Hosseinzadeh F, Verdi J, Ai J, et al (2018). Combinational immune-cell therapy of natural killer cells and sorafenib for advanced hepatocellular carcinoma: a review. Cancer Cell Int, 18, 133.

Hsu M-H, Hsu S-M, Kuo Y-C, et al (2017). Treatment with low-dose sorafenib in combination with a novel benzimidazole derivative bearing a pyrolidine side chain provides synergistic anti-proliferative effects against human liver cancer. $R S C A d v, 7,16253-63$.

Khakoo AY, Pati S, Anderson SA, et al (2006). Human mesenchymal stem cells exert potent antitumorigenic effects in a model of Kaposi's sarcoma. J Exp Med, 203, 1235-47.

Iijima M, Fukino K, Adachi M, et al (2011). Sorafenib-associated hand-foot syndrome in Japanese patients. J Dermatol, 38, 261-6.

Jahan S, Kumar D, Kumar A, et al (2017). Neurotrophic factor mediated neuronal differentiation of human cord blood mesenchymal stem cells and their applicability to assess the developmental neurotoxicity. Biochem Biophys Res Commun, 482, 961-7.

Lacouture M, Reilly L, Gerami P, et al (2008). Hand foot skin reaction in cancer patients treated with the multikinase inhibitors sorafenib and sunitinib. Ann Oncol, 19, 1955-61.

Li T, Song B, Du X, et al (2013). Effect of bone-marrow-derived mesenchymal stem cells on high-potential hepatocellular carcinoma in mouse models: an intervention study. Eur $J$ 
Med Res, 18, 34.

Liu L, Cao Y, Chen C, et al (2006). Sorafenib blocks the RAF/ MEK/ERK pathway, inhibits tumor angiogenesis, and induces tumor cell apoptosis in hepatocellular carcinoma model PLC/PRF/5. Cancer Res, 66, 11851-8.

Lu Y-r, Yuan Y, Wang X-j, et al (2008). The growth inhibitory effect of mesenchymal stem cells on tumor cells in vitro and in vivo. Cancer Biol Ther, 7, 245-51.

Ohlsson LB, Varas L, Kjellman C, et al (2003). Mesenchymal progenitor cell-mediated inhibition of tumor growth in vivo and in vitro in gelatin matrix. Exp Mol Pathol, 75, 248-55.

Otsu K, Das S, Houser SD, et al (2009). Concentration-dependent inhibition of angiogenesis by mesenchymal stem cells. Blood, 113, 4197-205.

Peng Y, Li Z, Yang P, et al (2014). Direct contacts with colon cancer cells regulate the differentiation of bone marrow mesenchymal stem cells into tumor associated fibroblasts. Biochem Biophys Res Commun, 451, 68-73.

Qiao L, Xu Z-1, Zhao T-j, et al (2008a). Dkk-1 secreted by mesenchymal stem cells inhibits growth of breast cancer cells via depression of Wnt signalling. Cancer lett, 269, 67-77.

Qiao L, Xu Z, Zhao T, et al (2008b). Suppression of tumorigenesis by human mesenchymal stem cells in a hepatoma model. Cell Res, 18, 500.

Sadaf N, Kumar N, Ali M, et al (2018). Arsenic trioxide induces apoptosis and inhibits the growth of human liver cancer cells. Life Sci, 205, 9-17.

Sage EK, Thakrar RM, Janes SM (2016). Genetically modified mesenchymal stromal cells in cancer therapy. Cytotherapy, 18, 1435-45.

Strumberg D, Clark JW, Awada A, et al (2007). Safety, pharmacokinetics, and preliminary antitumor activity of sorafenib: a review of four phase I trials in patients with advanced refractory solid tumors. Oncologist, 12, 426-37.

Wu N, Zhang Y-L, Wang H-T, et al (2016). Overexpression of hepatocyte nuclear factor $4 \alpha$ in human mesenchymal stem cells suppresses hepatocellular carcinoma development through Wnt/B-catenin signaling pathway downregulation. Cancer Biol Ther, 17, 558-65.

Yoo HY, Patt CH, Geschwind J-F, et al (2003). The outcome of liver transplantation in patients with hepatocellular carcinoma in the United States between 1988 and 2001: 5 -year survival has improved significantly with time. J Clin Oncol, 21, 4329-35.

Youssef MM, Tolba MF, Badawy NN, et al (2016). Novel combination of sorafenib and biochanin-A synergistically enhances the anti-proliferative and pro-apoptotic effects on hepatocellular carcinoma cells. Sci Rep, 6, 30717.

\section{c) (i) (8)}

This work is licensed under a Creative Commons AttributionNon Commercial 4.0 International License. 